\title{
ANNIHILATOR ALTERNATIVE ALGEBRAS
}

\author{
I. P. DE GUZMAN
}

The aim of this paper is to use in the alternative case the axioms of the annihilator Banach algebras, and obtain some structure theorems similar to the known ones in the associative case.

1. Introduction. As corollary of the main theorem of this paper: Theorem 3.11, we prove (see Corollary 3.12) that if $A$ is a Kleinfeld semisimple annihilator complex purely-alternative algebra, then $A$ is the closure of the direct sum of all its ideals which are isomorphic to the algebra of complex octonions $\theta_{\mathbf{C}}$.

2. Prerequisites and notation. In this paper, $A$ is always understood to be an alternative algebra. "Ideal" without further qualification will mean "ideal of $A$ ".

(2.1) $N(A)$ denote the nucleus of $A$ (for the definition of $N(A)$, see for example [3]).

We say an ideal (or right ideal or left ideal) $I$ is nuclear provided $\{0\} \neq I \subset N(A)$.

(2.2) In what follows we write $D_{0}$ for the associator ideal of $A$, and $U_{0}$ its maximun nuclear ideal. It is known that $U_{0} D_{0}=D_{0} U_{0}=\{0\}$ (see [6]).

(2.3) If $I$ is an ideal, and $I \cap D_{0}=\{0\}$, then $I \subset U_{0}$. A proof is easy.

(2.4) We say an alternative algebra is purely-alternative provided $U_{0}=\{0\}$ (See [6]).

(2.5) Say a right ideal $I$ of $A$ is trivial provided $I \neq\{0\}=I^{2}$, and say that $A$ is semiprime provided $A$ has no trivial right ideal.

$A$ is semiprime if and only if $A$ contains no trivial ideal. (For a proof see [7]).

(2.6) For each right ideal $R, \operatorname{Lan}(R)$ denotes the left annihilator of $R$ : $\operatorname{Lan}(R)=\{a \in A: a R=\{0\}\}$. If $L$ is a left ideal, $\operatorname{Ran}(L)=\{a \in A$ : $L a=\{0\}\}$ is the right annihilator of $L$. If $I$ is a right ideal of an ideal $B$ of $A$, we note by $\operatorname{Lan}_{B}(I)$ the left annihilator of $I$ in the algebra $B$.

(2.7) An element $u$ of an algebra $A$ is a right modular unit for a vector subspace $E$ of $A$ if $\{a-a u: a \in A\} \subset E$.

A modular left ideal is a left ideal for which there exist a right modular unit. 
(2.8) An alternative algebra $A$ is said primitive in case it contains a maximal modular left ideal which contains no ideal of the algebra other that the zero ideal.

(2.9) An ideal $P$ of $A$ is said primitive if $A / P$ is a primitive algebra.

(2.10) We define the Kleinfeld Radical of an alternative algebra $A$ as the algebra, if there are no primitive ideals, and the intersection of all primitive ideals in the algebra if there are such ideals.

The algebra is said to be Kleinfeld semisimple if the Kleinfeld Radical is $\{0\}$.

We shall denote the Kleinfeld Radical of $A$ by $\operatorname{Rad}_{K}(A)$. The Kleinfeld Radical is actually a ideal of $A$.

(2.11) If $A$ is a Kleinfeld semisimple alternative algebra, $A$ is semiprime. A proof is easy.

(2.12) An alternative algebra $A$ is called simple in case $\{0\}$ and $A$ itself are the only ideals of $A$ and $A^{2} \neq\{0\}$.

(2.13) The notions of simple algebra and simple ring coincide. (For a proof see [5]).

(2.14) Let $A$ be an alternative algebra which is not associative. Then the following are equal:

(i) $A$ is a simple algebra

(ii) $A$ is a primitive algebra

(iii) $A$ is a Cayley-Dickson algebra over its centre.

Proof. If $A$ is simple algebra, then $A$ is a simple ring (2.13), it follows by [8], Theorem $\mathrm{C}$, that $A$ is a Cayley-Dickson algebra over its centre. Now let $A$ be a primitive algebra, we can apply the arguments of [4] Theorem 2 to prove that $A$ is a simple algebra. Finally any Cayley-Dickson algebra is simple, [5] p. 48.

(2.15) Since $\mathbf{C}$ is algebraically closed, there is one and (up to isomorphism) only one Cayley-Dickson algebra over $\mathbf{C}$ ([5], p.53). This algebra is called the algebra of complex octonions and denoted $\theta_{\mathbf{C}}$.

3. Annihilator alternative algebras. We shall use concepts and nomenclature of the classic theory of normed associative algebras taking as standard reference [1].

Definition 3.1. A complete normed alternative algebra $A$ is said to be an annihilator alternative algebra if it satisfies the following axioms: for all closed left ideals $L$ and all closed right ideals $R$,

(i) $\operatorname{Ran}(L)=\{0\}$ if and only if $L=A$.

(ii) $\operatorname{Lan}(R)=\{0\}$ if and only if $R=A$. 
Proposition 3.2. Every not associative, alternative normed primitive complex algebra $A$ is algebraically isomorphic to the algebra $\theta_{\mathbf{C}}$.

Proof. By (2.14) $A$ is an algebra of octonions over its center. But this center is a complex associative commutative normed division algebra.

LEMMA 3.3. Let $A$ be a semiprime annihilator alternative algebra, let $B$ be an ideal of $A$. Then,

(i) $\operatorname{Lan}(B)=\operatorname{Ran}(B)$ is an ideal of $A$.

(ii) $B \cap \operatorname{Lan}(B)=\{0\}$.

(iii) $\overline{B \oplus \operatorname{Lan}(B)}=A$.

Proof. A proof of (i) is easy. Now we can apply the proof of the associative case ([1], §32, Lemma 4).

LEMMA 3.4. Let $A$ be a semiprime annihilator alternative algebra, let $B$ be a closed ideal of $A$, and let $L$ be a closed left ideal of $B$. Then,

(i) $L$ is a closed left ideal of $A$.

(ii) $B$ is a semiprime complete normed alternative algebra.

(iii) If $\operatorname{Ran}(L) \cap B=\{0\}$, then $B A \subset L$ and $\operatorname{Ran}(L)=\operatorname{Lan}(L)=$ $\operatorname{Ran}(B)$.

Proof. Bonsall's proof [1], §32, Lemma 8 applies word for word taking into account Lemma 3.3.

Proposition 3.5. Let $A$ be a semiprime annihilator alternative algebra and let $B$ be a closed ideal with $\overline{A B}=\overline{B A}=B$. Then $B$ is a semiprime annihilator alternative algebra.

Proof. By 3.4 (ii), $B$ is a semiprime. Now we can apply the proof of the associative case $([1], \S 32$, Theorem 9) taking account Lemma 3.3.

Proposition 3.6. Let $A$ be a semiprime alternative algebra. Then,

(i) If $I, J$ are ideals of $A$ such that $A=I \oplus J$, then $I=\operatorname{Lan}(J)$

(ii) If, in addition, $A$ is normed. Then, $I, J$ are closed ideals and if $A$ is complete normed, the sum in ( $i)$ is direct

(iii) If, in addition, $A$ is annihilator. Then, $I, J$ are semiprime annihilator alternative algebras.

Proof. (i) $I J \subset I \cap J=\{0\}$ implies that $I \subset \operatorname{Lan}(J)$. On the other hand, let $a$ be, $a \in \operatorname{Lan}(J), a=x+y$ with $x \in I$ and $y \in J$. Then, 
$\{0\}=a J=x J+y J=y J$. Therefore $y \in J \cap \operatorname{Lan}(J)=\{0\} \quad$ (3.3(ii)). Thus $\operatorname{Lan}(J) \subset I$. This completes the proof of (i).

(ii) Clearly.

(iii) By (ii) and 3.4(ii) $I, J$ are semiprime complete normed alternative algebras.

We prove next that $I, J$ are annihilator. Let $L$ be a closed left ideal of $J$, by Lemma 3.4(i) $L$ is a closed left ideal of $A$.

$\operatorname{Ran}_{I}(L)=\operatorname{Ran}(L) \cap I=\{0\}$ implies by 3.4(iii) that $\operatorname{Ran}(L)=$ $\operatorname{Ran}(I)$. Suppose that $L \neq I$. Then $L \oplus \operatorname{Ran}(L)=L \oplus \operatorname{Ran}(I) \neq A$, therefore $\{0\} \neq \operatorname{Ran}(L \oplus \operatorname{Lan}(L))=\operatorname{Ran}(L) \cap \operatorname{Ran}(\operatorname{Lan}(L))$, which is impossible by Lemma 3.3(ii). Therefore $L=I$. Analogically if $R$ is a closed right ideal of $J$.

Proposition 3.7. Let $A$ be a semiprime annihilator alternative algebra, let $B$ be a closed ideal of $A$, and suppose that the left regular representation of $B$ on $B$ is a homeomorphism. Then $A=B \oplus \operatorname{Lan}(B)=B \oplus \operatorname{Ran}(B)$. Moreover $B$ and $\operatorname{Lan}(B)$ are semiprime annihilator alternative algebras.

Proof. Bonsall's proof [1], §32, Proposition 14, applies word for word taking into account Lemma 3.3 and above proposition.

Note. The Proposition 3.7 provides a first structure theorem for semiprime annihilator alternative algebras.

Proposition 3.8. Let $A$ be a semiprime annihilator alternative algebra. Then,

(i) $U_{0}=\operatorname{Lan}\left(D_{0}\right)$.

(ii) $A$ is purely-alternative if and only if $A=\bar{D}_{0}$.

Proof. (i) By (2.2), $U_{0} \subset \operatorname{Lan}\left(D_{0}\right)$. On the other hand, $D_{0} \cap \operatorname{Lan}\left(D_{0}\right)$ $=\{0\}$, therefore by (2.3), $\operatorname{Lan}\left(\bar{D}_{0}\right) \subset \bar{U}_{0}=U_{0}$, and so, $U_{0}=\operatorname{Lan}\left(D_{0}\right)=$ $\operatorname{Lan}\left(\overline{D_{0}}\right)$.

(ii) $\{0\}=\operatorname{Lan}\left(\bar{D}_{0}\right)=U_{0}$ if and only if $A=\bar{D}_{0}$.

Corollary 3.9. Let $A$ be a semiprime annihilator alternative algebra. Then, $A=\overline{U_{0} \oplus \overline{D_{0}}}$.

Proof. Proposition 3.8(i) and Lemma 3.3(iii), taking account that $U_{0}$ is closed. 
THEOREM 3.10. Let $A$ be a semiprime annihilator complex alternative algebra. Then,

(i) If $P$ is a primitive ideal of $A$ such that $D_{0} \not \subset P$, there exists exactly an ideal $N$ of $A$ which is isomorphic to the algebra $\vartheta_{\mathbf{C}}$ and $A=P \oplus N$.

(ii) If $N$ is an ideal of $A$ which is isomorphic to the algebra $\theta_{C}$, there exists exactly one primitive ideal $P$ of $A$ such that $D_{0} \not \subset P$, and $A=P \oplus N$.

Proof. (i) If $P$ is a primitive ideal such that $D_{0} \not \subset P$, then $A / P$ is a primitive normed alternative complex algebra which is not associative. It follows by proposition 3.2, that it is isomorphic to $\theta_{\mathbf{C}}$ and $P$ is of codimension 8. If we note $N=\operatorname{Lan}(P)$, by Lemma $3.3 A=\overline{P \oplus N}$. Since $P \cap N=\{0\}$, we have that $N$ has finite dimension and so $P+N$ is a closed ideal, therefore $A=P \oplus N$ and $N$ is isomorphic to $\theta_{\mathrm{C}}$.

(ii) If $N$ is an ideal of $A$ isomorphic to $\theta_{\mathbf{C}}, N$ has finite dimension. Now we can apply Proposition 3.7, if we note $P=\operatorname{Lan}(N)$ we have $A=N \oplus P$, since $A / P$ is isomorphic to $\theta_{C}$. It follows by (2.9) that $P$ is a primitive ideal.

THEOREM 3.11. Let $A$ be a semiprime annihilator complex alternative algebra and let $\{I: \alpha \in \Lambda\}$ the collection of all its ideals which are isomorphic to the algebra $\theta_{\mathbf{C}}$. Then,

(i) If $B$ is the closure of the direct sum of the ideals of this collection, $B$ is a semiprime annihilator purely-alternative algebra

(ii) If, in addition, $A$ is Kleinfeld semisimple, then $\operatorname{Lan}(B)=U_{0}$ and $A=\overline{B \oplus \operatorname{Lan}(B)}$.

Proof. (i) Let $R=\Sigma_{\alpha \in \Lambda} I_{\alpha}$. If $\alpha \neq \beta, I_{\alpha} I_{\beta}$ is an ideal of $A$ and therefore, an ideal of $I_{\alpha}$ and of $I_{\beta}$. Since $I_{\alpha}, I_{\beta}$ are simple algebras, we have that $I_{\alpha} I_{\beta}=\{0\}$ and the sum is direct.

Now let $e_{\alpha}$ be the unit of $I_{\alpha}$. Since

$$
a_{\alpha_{1}}+, \ldots,+a_{\alpha_{k}}=\left(e_{\alpha_{1}}+, \ldots,+e_{\alpha_{k}}\right)\left(a_{\alpha_{1}}+, \ldots,+a_{\alpha_{k}}\right),
$$

we have $R \subset R^{2}$ and therefore $R=R^{2}$, thus $\overline{R^{2}}=\bar{R}=B$ and $\overline{B^{2}}=B$.

Finally, $B=\overline{B^{2}} \subset \overline{A B}$ and $\overline{A B} \subset B$, thus $B=\overline{A B}$. Analogically $B$ $=\overline{B A}$ By Proposition 3.5, $B$ is a semiprime annihilator algebra.

Now, in view of that each ideal $I_{\alpha}$ is a simple algebra, its associator ideal coincides with $I_{\alpha}$, therefore, if $D_{B}$ denote the associator ideal of the algebra $B$, we have, $R \subset D_{B} \subset D_{0}$ and $B=\bar{R} \subset \overline{D_{B}} \subset B$, thus $B=\overline{D_{B}}$ and Corollary 3.9 show that $B$ is purely-alternative.

(ii) Suppose additionally that $A$ is Kleinfeld semisimple. Let $H$ be the set of all those ideals $P$ of $A$ such that $P$ is a primitive ideal, if we write 
$H=H_{1} \cup H_{2}$ where $H_{1}$ is the set of all those ideals $P$ of $H$ such that $D_{0} \not \subset P$ and $H_{2}$ is the set of all those ideals $P$ of $H$ such that $D_{0} \subset P$, then $\operatorname{Lan}(B)=\operatorname{Lan}(R)=\bigcap_{\alpha \in \Lambda} \operatorname{Lan}\left(I_{\alpha}\right)$ and by Theorem 3.10, $\operatorname{Lan}(B)=$ $\cap_{P \in H_{1}} P$.

On the other hand, given $m \in \operatorname{Lan}(B)$ and $a, b \in A$ we have $(m, a, b) \in \operatorname{Lan}(B)=\bigcap_{P \in H_{1}} P$ and, clearly, $(m, a, b) \in \bigcap_{P \in H_{2}} P$. Whence $(m, a, b) \in \bigcap_{P \in H} P=\operatorname{Rad}_{K}(A)=\{0\}$. So $\operatorname{Lan}(B) \subset U_{0}$, and by Proposition 3.8, $\operatorname{Lan}(B) \subset U_{0} \subset \operatorname{Lan}\left(D_{0}\right) \subset \operatorname{Lan}\left(D_{B}\right)=\operatorname{Lan}\left(\overline{D_{B}}\right)$. But $\overline{D_{B}} \subset B$, and so $\operatorname{Lan}(B)=U_{0}$ and $A=\overline{B \oplus \operatorname{Lan}(B)}$.

COROLlaRY 3.12. If $A$ is a Kleinfeld semisimple annihilator complex purely-alternative algebra, then $A$ is the closure of the direct sum of all its ideals which are isomorphic to the algebra $\theta_{\mathbf{C}}$.

Proof. Theorem 3.11 and Proposition 3.8

\section{REFERENCES}

1. F. F. Bonsall, and J. Duncan, Complete Normed Algebras, Springer-Verlag, Berlin, Heidelberg, New York, (1973).

2. F. F. Bonsall, and A. W. Goldie, Annihilator algebras, Proc. London Math. Soc., (3) 4, (1954), 154-167.

3. R. H. Bruck, and E. Kleinfeld, The structure of alternative division rings, Proc. Am. Math. Soc., 2 (1951), 878-890.

4. E. Kleinfeld, Primitive alternative rings and semisimplicity, Amer. J. Math., 77 (1955), $725-730$.

5. R. D. Schafer, An Introduction to Non Associative Algebras, Academic Press, New York, San Francisco, London (1966).

6. M. Slater, Nucleus and center in alternative rings, J. Algebra, 7 (1967), 372-388.

$7 . \quad$ Ideals in semiprime alternative rings, J. Algebra, 8 (1968), 60-76.

$8 . \quad$ _ The open case for simple alternative rings, Proc. Amer. Math. Soc., 19 (1968), $712-715$.

Received June 3, 1981.

UNIVERSIDAD DE MALAGA

SPAIN (Malaga-7) 